\title{
Can proxy assessments serve as a first screener for identifying people at risk for multidimensional frailty?
}

\author{
Anne van der Vorst ${ }^{1}$ (D) G. A. Rixt Zijlstra ${ }^{1}$ - Nico De Witte ${ }^{2,3} \cdot$ Jan De Lepeleire ${ }^{4}$ - Gertrudis I. J. M. Kempen ${ }^{1}$ \\ Jos M. G. A. Schols ${ }^{1,5} \cdot$ D-SCOPE Consortium
}

Received: 9 January 2018 / Accepted: 8 May 2018 / Published online: 24 May 2018

(c) The Author(s) 2018

\begin{abstract}
Purpose Timely detection of multidimensional frailty is important to prevent further negative outcomes. Perspectives of general practitioners (GPs) or informal caregivers might serve as a first, global screener to identify older people in need of a more extended assessment. Therefore, we aimed to investigate whether proxy assessments are associated with older people's self-reported environmental, physical, psychological, social and overall frailty.

Methods A cross-sectional study was conducted on 78 community-dwelling people aged 60 years and over, their GPs $(n=57)$ and informal caregivers $(n=50)$. Self-reported frailty was assessed with the Comprehensive Frailty Assessment Instrument. GPs and informal caregivers rated each frailty domain and overall frailty on a scale of 0 (not frail at all) to 10 (severely frail). Associations between proxy scores and self-reported frailty were examined by correlation analyses.

Results Significant low to moderate associations were found between (1) self-reported physical frailty and physical frailty scores given by the GPs $(r=0.366, p \leq 0.01)$ and informal caregivers $(r=0.305, p \leq 0.05)$, and (2) self-reported psychological frailty and psychological frailty scores given by the GPs $(r=0.230, p \leq 0.05)$ and informal caregivers $(r=0.254, p \leq 0.05)$. No significant associations were found between proxy scores and self-reported environmental, social and overall frailty. Conclusions Global proxy scores as short, subjective screeners for detecting frailty cannot completely replace self-reported frailty. Nonetheless, low to moderate correlations were found for physical and psychological frailty ratings, suggesting that proxy scores might be of value as a first sign of something being wrong for these domains.
\end{abstract}

Keywords Cross-sectional studies · Informal caregivers · General practitioners · Independent living · Outcome assessment

Membership of the D-SCOPE Consortium is provided in the Acknowledgments.

Anne van der Vorst

a.vandervorst@maastrichtuniversity.nl

G. A. Rixt Zijlstra

r.zijlstra@maastrichtuniversity.nl

Nico De Witte

nico.dewitte@hogent.be

Jan De Lepeleire

jan.delepeleire@kuleuven.be

Gertrudis I. J. M. Kempen

g.kempen@maastrichtuniversity.nl

Jos M. G. A. Schols

jos.schols@maastrichtuniversity.nl

\section{Introduction}

Although frailty is often seen as merely a physical construct [1], it can also be regarded as a multidimensional phenomenon that includes experiencing losses in physical,

1 Department of Health Services Research, Care and Public Health Research Institute (CAPHRI), Maastricht University, P.O. Box 616, 6200 MD Maastricht, The Netherlands

2 Faculty of Psychology and Educational Sciences, Vrije Universiteit Brussel, Brussels, Belgium

3 Faculty of Education, Health and Social Work, University College Ghent, Ghent, Belgium

4 Department of Public Health and Primary Care, University of Leuven, Louvain, Belgium

5 Department of Family Medicine, Care and Public Health Research Institute (CAPHRI), Maastricht University, Maastricht, The Netherlands 
psychological, social, and environmental aspects [2]. Depending on the definition and measure used, prevalence rates of frailty vary between 4.2 and $59.1 \%$ [3]. Recent studies have shown that multidimensional frailty is associated with higher age [4], amongst other factors, and may lead to negative consequences such as disability and increased health care utilisation [5]. Therefore, frailty might jeopardise the wish of older people to 'age in place' [6]. This is also important from a policy perspective, for example, in terms of reducing the costs of long-term care [7]. In view of the larger proportion of older people in society today [8], and the possible negative consequences [5], timely detection of frailty is important for prevention strategies to reduce the risk of negative outcomes.

In recent years, various detection instruments for frailty have been developed, including self-report measures and performance-based assessments [9]. However, a short first screener, to indicate whether such a more extended assessment is needed, might be favorable [10]. Herewith, the perspective of formal and informal caregivers might be useful, especially within the context of 'ageing in place'. First, frail older people are likely to become one of the largest groups to visit their GP, with GP consultation rates at their highest for older age groups [11]. However, about $20 \%$ of community-dwelling frail older people do not have regular contact with their GP [12]. In addition, informal caregivers reported that GPs merely focus on medical aspects [13], which is not in line with our multidimensional approach. Therefore, it might be important to assess the perspective of informal caregivers as well. Of those aged $\geq 75$ years, $18.0 \%$ received informal care in 2015 [14], and it is expected that the proportion of people aged $\geq 65$ years to receive informal care will increase to $25 \%$ by 2030 [15]. Thus, it seems likely that GPs' and/or informal caregivers' perspectives could serve as a simple, short screener to indicate whether a more extended assessment is needed [10].

Some researchers have examined proxy assessments of frailty and compared them with objective measures such as Fried's criteria [16]. However, these studies did not focus on different frailty domains, nor did they compare proxy assessments with self-reported frailty. Hoogendijk and colleagues [17] did compare a multidimensional frailty assessment by a GP with a self-report measure. Their findings revealed some apparent differences between the two: a prevalence rate of multidimensional frailty of $28.6 \%$ according to the GPs, compared with $36.4 \%$ of the older people perceiving themselves to be frail based on the Groningen Frailty Indicator [17]. However, discrepancies between ratings on an individual level are unknown and ratings were not specified for different domains of frailty. Lastly, to the best of our knowledge, previous research has not investigated proxy assessments of multidimensional frailty by informal caregivers.
Therefore, the aim of this study was to assess whether GPs and informal caregivers can make reliable estimations about the level of frailty as perceived by the older adult, which then could serve as a first, global screener. We compared GPs' and informal caregivers' perspectives of the frailty level of the older adult, by asking simple, short, questions, with self-reported, multidimensional frailty. Based on previous research, it was hypothesized that agreement levels would be higher for observable aspects $[18,19]$, such as environmental and physical frailty, and lower for subjective aspects $[18,19]$, such as psychological and social frailty.

\section{Material end methods}

\section{Study design and participants}

Data from the cross-sectional D-SCOPE study (Detection, Support and Care for frail older people: Prevention and Empowerment) were used. 121 community-dwelling people aged 60 years and over were recruited via six healthcare organizations in Flanders, Belgium. Purposive sampling was used to over-sample people at risk for frailty [4], in addition to snowball sampling. Participants were interviewed in their home between November 2015 and April 2016 by trained interviewers. Afterwards, participants were asked whether their GP and/or informal caregiver could be invited for a proxy interview. However, 52 GPs were not interviewed: 1 older adult did not have a GP $(0.8 \%), 12$ refused an assessment by their GP (9.9\%), 16 GPs could not be reached during the study period (13.2\%) and 23 GPs did not want to participate (19.0\%). In addition, for 62 older people no informal caregivers were interviewed because 33 respondents did not have an informal caregiver (27.3\%), 17 refused a proxy assessment by their informal caregiver (14.0\%), 9 informal caregivers could not be reached during the study period (7.4\%) and 3 refused to participate (2.5\%). In the current study, older people were only included if they and at least one of their proxies completed the frailty measures. The final sample consisted of 78 older people, 57 GPs and 50 informal caregivers ( 8 older people did not complete the self-reported frailty measure; 12 GPs and 9 informal caregivers did not complete each domain of the frailty measure).

Informed consent was obtained from all older people, and the Ethics Committee on Human Sciences of the Vrije Universiteit Brussel approved the study (ECHW_031).

\section{Measures}

\section{Sample characteristics}

The following socio-demographic characteristics of the older people were assessed: age, gender, place of birth, marital 
status and living arrangement. Additionally, cognition was assessed with the Montreal Cognitive Assessment (MoCA) which measures, amongst others, attention, memory and orientation [20]. Scores were corrected for educational effects by giving one extra point to those with $\leq 12$ years of education [21]. Whereas the 'original' cut-off of $<26$ has been found to lead to a high number of false positives [22, 23], a cut-off of $<24$ has been proposed to indicate mild cognitive problems [22, 23]; the latter was applied in the present study. Age, gender and type of practice were assessed for the GPs, and age, gender, nationality, place of birth, marital status, living arrangement and relationship with the older adult for the informal caregivers.

\section{Frailty}

Self-reported multidimensional frailty was assessed using the validated Comprehensive Frailty Assessment Instrument (CFAI; [2]), which measures overall frailty and its subdomains environmental, physical, psychological and social frailty (e.g., 'My house is in a bad condition/poorly kept'; 'Have you been hampered in less demanding activities like carrying shopping bags by your state of health?'; 'I feel unhappy and depressed'; and 'There are enough people whom I can rely on when I am in trouble', respectively). Scores on each scale range from 0 to 25 , while overall frailty ranges from 0 to 100 . Higher scores indicate more severe levels of frailty, and cut-offs for high frailty per domain and for overall frailty have been determined based on two-step cluster analyses (De Witte et al., available upon request). Cut-off scores for high frailty per domain are as follows: 7.51 for environmental; 18.81 for physical; 11.51 for psychological; 16.01 for social; and 51.88 for overall frailty.

Proxies were asked to determine the level of frailty of the older person per domain and for overall frailty on a tenpoint scale [e.g., 'On a scale ranging from 0 to 10, how frail do you think (name of older adult) is regarding his/ her environment (e.g., adaptation of the home, evaluation of the neighbourhood), ranging from "not frail at all" (0) to "severely frail" (10)?']. No exact cut-offs for frailty were calculated. This rating is suggested as a first step in identifying frailty to indicate whether a more comprehensive assessment is needed [10].

\section{Statistical analyses}

First, prevalence rates of high frailty according to the CFAI were calculated per domain as well as for overall frailty, based on the cut-offs as defined by De Witte et al. (available upon request). Second, self-reported frailty was transformed into scores with a range of 0-10 (rather than 0-25 or $0-100$, respectively) to facilitate interpretation and comparisons with proxy scores. Third, descriptive statistics were conducted to characterise the sample. Fourth, normality of the data was checked. $Z$ scores for skewness and kurtosis were obtained and, due to the sample size $(>50)$, variables with $z$ scores $>3.29$ were classified as non-normal [24]. Thereafter, Chi-square (categorical data) and independent samples $t$ tests were performed to explore differences in socio-demographics (age, gender, marital status, living arrangement), cognitive functioning (MoCA) and selfreported frailty scores between those included and excluded from the current study. Lastly, correlations between proxy scores and self-reported frailty were computed using onetailed Pearson correlation. Statistical significance was set at $p<0.05$. All statistical analyses were carried out using IBM SPSS Statistics, Version 24 (SPSS Inc., Chicago, IL, USA).

\section{Results}

The older people included in the current study $(n=78)$ did not significantly differ from those excluded $(n=43)$ in terms of age, MoCA scores, self-reported frailty and gender. However, those included in this study were more often living with their partner (38.5 versus $18.6 \% ; p=0.024$ ) or married (37.2 versus $14.0 \%$; $p=0.001$ ).

\section{Sample characteristics}

The mean age of the older people was 80.0 years (SD 8.2; range 61-95), and 60.3\% were female. Mean MoCA score was 21.1 (SD 4.7, range 10-29). Out of 69 participants who completed the MoCA, $65.2 \%$ scored $<24$, indicating mild cognitive problems. Participating GPs had a mean age of 56.4 years (SD 8.5; range 26-78). The majority were male (73.7\%) and solo-practitioners (64.9\%). Informal caregivers had a mean age of 61.8 years (SD 13.8; range 28-92), 72.0\% were female and most were a child (in-law) of the older person $(58.0 \%)$. More sample characteristics are shown in Table 1.

\section{Self-reported multidimensional frailty and proxy scores}

The lower part of Table 1 shows mean self-reported frailty scores. Scores ranged from 1.7 to 4.8, with lowest scores for environmental frailty and highest for physical frailty. According to these self-reports, $14.1 \%$ of the respondents were environmentally frail, $19.2 \%$ physically, $20.5 \%$ psychologically, $12.8 \%$ socially and $30.8 \%$ overall (not tabulated).

Tables 2 and 3 show the mean scores and standard deviations for the frailty domains, and the correlations between self-reported frailty and proxy scores. Proxy scores ranged from 2.6 to 5.1, with lowest scores for environmental frailty and highest for physical frailty. There was a significant, 
Table 1 Sample characteristics

\begin{tabular}{|c|c|c|c|}
\hline & Older people $(n=78)$ & GPs $(n=57)$ & $\begin{array}{l}\text { Informal } \\
\text { caregivers } \\
(n=50)\end{array}$ \\
\hline \multicolumn{4}{|l|}{ Sample characteristics } \\
\hline \multicolumn{4}{|l|}{ Sociodemographics } \\
\hline Age (mean, SD) & $80.0(8.2)^{\mathrm{a}}$ & $56.4(8.5)^{\mathrm{b}}$ & $61.8(13.8)$ \\
\hline Females $(\%)$ & 60.3 & 26.3 & 72.0 \\
\hline Migration background (\%) & 15.4 & - & 2.0 \\
\hline \multicolumn{4}{|l|}{ Current marital status (\%) } \\
\hline Married & 37.2 & - & 66.0 \\
\hline Divorced & 5.1 & - & 14.0 \\
\hline Widowed & 52.6 & - & 6.0 \\
\hline Other & 5.1 & - & 14.0 \\
\hline Living alone (\%) & 52.6 & - & - \\
\hline \multicolumn{4}{|l|}{ Cognition } \\
\hline MoCA (mean, SD) & $21.1(4.7)^{\mathrm{c}}$ & - & - \\
\hline \multicolumn{4}{|l|}{ Practice $(\%)$} \\
\hline Solo & - & 64.9 & - \\
\hline Duo & - & 12.3 & - \\
\hline Group & - & 22.8 & - \\
\hline \multicolumn{4}{|c|}{ Relationship with older adult (\%) } \\
\hline Partner & - & - & 24.0 \\
\hline Child (in-law) & & - & 58.0 \\
\hline Grandchild & - & - & 2.0 \\
\hline Other family member & - & - & 8.0 \\
\hline Neighbor & - & - & 8.0 \\
\hline \multicolumn{4}{|l|}{ Frailty assessment } \\
\hline \multicolumn{4}{|l|}{ Frailty (mean, SD) } \\
\hline Environmental & $1.7(1.6)$ & & \\
\hline Physical & $4.8(3.5)$ & & \\
\hline Psychological & $2.6(2.4)$ & & \\
\hline Social & $3.9(1.9)$ & & \\
\hline Overall & $3.3(1.5)$ & & \\
\hline
\end{tabular}

Note. $\mathrm{MoCA}=$ Montreal Cognitive Assessment (range 0-30). Frailty: measured with the Comprehensive Frailty Assessment Instrument. Higher scores indicate higher levels of frailty (range 0-10)

${ }^{\mathrm{a}} n=77$

${ }^{\mathrm{b}} n=55$

${ }^{\mathrm{c}} n=69$

Table 2 Pearson correlations between older people's and GPs' frailty assessments $(n=57)$

\begin{tabular}{llll}
\hline & $\begin{array}{l}\text { Older people } \\
\text { (mean, SD) }\end{array}$ & GPs (mean, SD) & $\begin{array}{l}\text { Correlation }(r, p \\
\text { value) }\end{array}$ \\
\hline Frailty & & & \\
Environmental & $1.5(1.5)$ & $2.6(2.2)$ & $0.093(0.245)$ \\
Physical & $4.8(3.3)$ & $4.2(2.4)$ & $0.366(0.003)$ \\
Psychological & $2.7(2.3)$ & $3.6(2.7)$ & $0.230(0.043)$ \\
Social & $3.6(1.8)$ & $3.0(2.6)$ & $0.177(0.094)$ \\
Overall & $3.2(1.4)$ & $4.2(2.6)$ & $0.141(0.148)$ \\
\hline
\end{tabular}

Note. Frailty: higher scores indicate higher levels of frailty (range 0-10). Older people: frailty measured with the Comprehensive Frailty Assessment Instrument; proxies: 1-item questions moderate relationship between self-reported frailty and GPs' scores for physical frailty $(r=0.366, p \leq 0.01)$, and a low correlation similarly for psychological frailty $(r=0.230$, $p \leq 0.05$ ). In addition, a significant, moderate relationship between informal caregivers' scores of physical frailty and self-reported physical frailty, $(r=0.305, p \leq 0.05)$, and a low correlation similarly for psychological frailty $(r=0.254, p \leq 0.05)$ were found. No significant correlations were found for environmental, social or overall frailty.

After conducting correlation analyses separately for those aged 80 years and over $(n=39)$, and 79 years or younger $(n=36)$, differences in correlations were observed. Only in those aged 80 years and over a significant association 
Table 3 Pearson correlations between older people's and informal caregivers' frailty assessments $(n=50)$

\begin{tabular}{llll}
\hline & $\begin{array}{l}\text { Older people } \\
(\text { mean, SD) }\end{array}$ & $\begin{array}{l}\text { Informal car- } \\
\text { egivers (mean, } \\
\text { SD) }\end{array}$ & $\begin{array}{l}\text { Correlation }(r, p \\
\text { value) }\end{array}$ \\
\hline Frailty & & & \\
$\quad$ Environmental & $1.8(1.7)$ & $2.6(0.7)$ & $0.121(0.202)$ \\
Physical & $4.7(3.7)$ & $5.1(2.2)$ & $0.305(0.016)$ \\
Psychological & $2.3(2.2)$ & $4.1(2.3)$ & $0.254(0.038)$ \\
Social & $3.9(1.9)$ & $3.5(2.7)$ & $0.027(0.427)$ \\
Overall & $3.3(1.6)$ & $4.7(2.4)$ & $0.127(0.189)$ \\
\hline
\end{tabular}

Note. Frailty: higher scores indicate higher levels of frailty (range $0-10)$. Older people: frailty measured with the Comprehensive Frailty Assessment Instrument; proxies: 1-item questions

between GPs' scores and self-reported physical frailty was found ( $r=0.663, p \leq 0.001$ ), as well as a borderline significant association between informal caregivers' scores and self-reported physical frailty $(r=0.296, p=0.056$ ) (findings not tabulated).

\section{Discussion}

This study assessed the associations between one-item proxy scores by GPs and informal caregivers, as a first screener, and self-reported multidimensional frailty in communitydwelling people aged 60 years and over. The results show that the relationship between proxy scores and self-reported frailty differs per domain, although proxy scores by GPs and informal caregivers were rather consistent. Whereas physical and psychological frailty scores by proxies and older people were significantly correlated, though only to a low (psychological frailty) to moderate (physical frailty) degree, no significant correlations were found for environmental, social and overall frailty. Thus, results suggest that proxy scores cannot completely replace self-reported multidimensional frailty. Nonetheless, for physical and psychological frailty, proxy scores might give an initial indication of whether further assessment is needed.

Our finding that physical frailty scores are moderately related might not be surprising as it corresponds with previous research on agreement levels $[19,25]$, and might be due to the observable nature of physical frailty $[18,19]$. The low correlations between psychological frailty scores are comparable with previous research as well [25]. Nonetheless, for physical frailty, proxy assessments seem particularly useful at higher ages, as correlations only remained significant in those aged 80 years and over. This might be explained by the well-known relationship between higher age and physical frailty [1].
However, despite the observable nature of environmental frailty, such as housing condition, self-reported and proxyassessed environmental frailty were not significantly related. This unexpected finding might be explained by proxies focusing on the need for adaptations in the house, in contrast to older people who prefer a home to which they are emotionally attached, even if it is 'suboptimal' [26]. Older people feel more purposeful when they are able to overcome difficulties, such as walking stairs [26]. Both could cause older people to self-report lower levels of environmental frailty.

Regarding social frailty, previous research has shown discrepancies between patient and GP scores on social aspects such as family relationships, possibly because these topics are not discussed during consultation [27]. However, while they also argued that assessments by family members are more similar to self-reports [27], we found no significant correlation with the scores of the informal caregivers either. Informal caregivers might feel that they are the ones on which the older people can rely when in difficulty, whereas older people might miss having people around them during the day.

However, other factors might explain our findings as well. First, proxies might have given a score based on their own perspective, rather than what they thought the older adult's perspective would be [28]. Second, frailty is still defined merely as a medical concept [29], which is not in line with our multidimensional approach. Third, sociodemographic characteristics might influence ratings, although findings on this differ between studies. While one study showed that neither gender, whether or not the patient and proxy live together, nor the closeness of the relationship influenced the association between scores [30], others argue that the amount of time spent together influences whether ratings are comparable [19], as does whether patients and proxies live together [31]. Fourth, people tend to rate themselves more favorably than is the case in reality [32] while proxies tend to report more difficulties [25], possibly due to higher levels of caregiver burden [25, 33]. Lastly, previous research has shown that formal and informal proxies are usually equally (in)correct [31], which seems in line with our findings.

\section{Strengths and limitations}

This study has several strengths. First, it is the first study to assess formal and informal proxy scores of environmental, physical, psychological, social and overall frailty. Second, our inclusion rate of proxy scores by GPs $(57.0 \%)$ lies within the range of other studies [34]. However, some limitations should be mentioned as well. First, different frailty measures were applied in the different populations. Consequently, scores by the older people and their proxies were not directly comparable. Because there are no cut-offs for the proxy 
measures available, the number of false positives and false negatives could not be determined. Nonetheless, as both scores ranged from 'not frail at all' to 'severely frail', it was possible to examine correlations. Second, participants had relatively low scores on the MoCA, and cognitive impairment could cause denial of problems or reduced awareness of problems [35]. However, it is known that many participants score below the cut-off in population-based cohorts, and it has been argued that this threshold should be lower [36]. Yet, there is no consensus about an optimal cut-off score so far [36]. Third, the sample size was relatively low, partly due to the high number of missing values (i.e., on the frailty measures or because no proxy assessments were conducted). However, significant differences between those included and excluded were only found for living arrangement and marital status, which might have merely influenced whether a spouse was available as an informal proxy.

\section{Conclusion and implications}

Proxy scores cannot be a substitute for self-reported frailty, although proxy scores of physical and psychological frailty might serve as a first step in the process of screening (i.e., as an indicator for the need of a more extended assessment). However, further research should be conducted to gain more insight into factors underlying these discrepancies.

First, future studies should include a larger number of older people and proxies, and should assess self-reported frailty with both a validated scale and a simple, one-item measure. In addition, factors that might influence correlation levels, such as contact frequency, should be taken into account. Furthermore, the potentially different perspectives of older people and their proxies could be investigated in more depth by means of a qualitative study. Moreover, longitudinal studies should take adverse outcomes into account, to be able to investigate the predictive value of proxy-assessed frailty per domain, and if this differs compared with selfreported frailty. Lastly, whereas we aimed to compare proxy assessments with self-reported frailty, for clinical practice it might be necessary to gain insight into multiple perspectives (i.e., from the older people as well as from proxies) to develop a richer understanding of the actual frailty level of the older person [29], given that frailty is a multidimensional and complex construct.

Acknowledgements The D-SCOPE Consortium is an international research group and is composed of researchers from University of Antwerp, Vrije Universiteit Brussel, University College Ghent, Catholic University of Leuven (Belgium), and Maastricht University (The Netherlands): Peter Paul De Deyn, Liesbeth De Donder, Jan De Lepeleire, Ellen De Roeck, Nico De Witte, Eva Dierckx, Daan Duppen, Sarah Dury, Sebastiaan Engelborghs, Bram Fret, Sylvia Hoens, Lieve Hoeyberghs, Tinie Kardol, Gertrudis I. J. M Kempen, Deborah Lambotte, Birgitte Schoenmakers, Jos M. G. A. Schols, An-Sofie Smetcoren, Lise Switsers, Michaël Van Der Elst, Anne van der Vorst, Dominique Verté,
G. A. Rixt Zijlstra. The authors would like to thank all participating older people, GPs, and informal caregivers.

Author contributions AvdV: conception and design of the study, acquisition of data, analysis and interpretation of data, drafting the article, final approval of this version to be submitted. RZ, NDW, JDL, GIJMK, and JMGAS: conception and design of the study, interpretation of data, revising the article and final approval of this version to be submitted.

Funding The study was funded by the Flemish government Agency for Innovation by Science and Technology, and is embedded in the Strategic Basic Research [Grant number IWT-140027-SBO]. The funders had no role in the study design; in the collection, analysis and interpretation of the data; in the writing of the report; nor in the decision to submit the article for publication.

\section{Compliance with ethical standards}

Conflict of interest The authors declare that they have no conflict of interest.

Ethical approval The Ethics Committee on Human Sciences of the Vrije Universiteit Brussel approved the study (ECHW_031).

Informed consent Informed consent was obtained from all older people.

Research Data Policy Data are available from the authors upon reasonable request.

Open Access This article is distributed under the terms of the Creative Commons Attribution 4.0 International License (http://creativeco mmons.org/licenses/by/4.0/), which permits unrestricted use, distribution, and reproduction in any medium, provided you give appropriate credit to the original author(s) and the source, provide a link to the Creative Commons license, and indicate if changes were made.

\section{References}

1. Fried LP, Tangen CM, Walston $\mathrm{J}$ et al (2001) Frailty in older adults: evidence for a phenotype. J Gerontol A Biol Sci Med Sci 56(3):146-157

2. De Witte N, Gobbens R, De Donder L et al (2013) The comprehensive frailty assessment instrument: development, validity and reliability. Geriatr Nurs 34(4):274-281

3. Collard RM, Boter H, Schoevers RA, Oude Voshaar RC (2012) Prevalence of frailty in a community-dwelling older persons: a systematic review. J Am Geriatr Soc 60(8):1487-1492

4. Dury S, De Roeck E, Duppen D et al (2017) Identifying frailty risk profiles of home-dwelling older people: focus on sociodemographic and socioeconomic characteristics. Aging Ment Health 21(10):1031-1039

5. Gobbens RJ, van Assen MALM, Luijkx KG, Schols JMGA (2012) The predictive validity of the Tilburg Frailty Indicator: disability, health care utilization, and quality of life in a population at risk. Gerontologist 52(5):619-631

6. Wiles JL, Leibing A, Guberman N, Reeve J, Allen RES (2012) The meaning of "aging in place" to older people. Gerontologist 52(3):357-366

7. Scharlach A (2011) Creating aging-friendly communities in the United States. Ageing Int 37(1):25-38 
8. European Commission (2014) The 2015 ageing report. http:// ec.europa.eu/economy_finance/publications/european_econo my/2014/pdf/ee8_en.pdf. Accessed 8 Dec 2016

9. Bouillon K, Kivimaki M, Hamer M et al (2013) Measures of frailty in population-based studies: an overview. BMC Geriatr. https://doi.org/10.1186/1471-2318-13-64

10. De Lepeleire J, Degryse J, Illiffe S, Mann E, Buntinx F (2008) Family physicians need easy instruments for frailty. Age Ageing 37:484-485

11. Wang Y, Hunt K, Nazareth I, Freemantle N, Petersen I (2013) Do men consult less than women? An analysis of routinely collected UK general practice data. BMJ Open. https://doi.org/10.1136/ bmjopen-2013-003320

12. van Campen C (ed) (2011) Kwetsbare ouderen. Sociaal en Cultureel Planbureau (in Dutch)

13. Cès S, Flusin D, Schmitz O et al (2016) Mantelzorgers van thuiswonende ouderen in België: een cruciale en complexe rol. Dataonderzoek. 2016 Koning Boudewijnstichting (in Dutch)

14. Verbeek-Oudijk D, Putman L, de Klerk M (2015) Zorg en ondersteuning in Nederland: kerncijfers. https://www.scp.nl/Publicatie s/Alle_publicaties/Publicaties_2017/Zorg_en_ondersteuning_in_ Nederland_kerncijfers_2015. Accessed 8 Feb 2017 (in Dutch)

15. Sadiraj K, Timmermans T, Ras M, de Boer A (2009) De toekomst van de mantelzorg. Sociaal en Cultureel Planbureau, Den Haag

16. Salter ML, Gupta N, Massie AB et al (2015) Perceived frailty and measured frailty among adults undergoing hemodialysis: a cross-sectional analysis. BMC Geriatr. https://doi.org/10.1186/ s12877-015-0051-y

17. Hoogendijk EO, Van Der Horst HE, Deeg DJ et al (2013) The identification of frail older adults in primary care: comparing the accuracy of five simple instruments. Age Ageing 42(2):262-265

18. McPherson CJ, Addington-Hall JM (2003) Judging the quality of care at the end of life: can proxies provide reliable information? Soc Sci Med 56(1):95-109

19. Snow AL, Cook KF, Lin PS, Morgan RO, Magaziner J (2005) Proxies and other external raters: methodological considerations. Health Serv Res 40:1676-1693

20. Nasreddine ZS, Phillips NA, Bédirian V et al (2005) The Montreal Cognitive Assessment, MoCA: a brief screening tool for mild cognitive impairment. J Am Geriatr Soc 53(4):695-699

21. Julayanont $P$, Hemrungrojn $S$, Tangwongchai S (2013) The effect of education and literacy on performance on the Montreal Cognitive Assessment among cognitively normal elderly. Alzheimers Dement 9(4):793

22. Luis CA, Keegan AP, Mullan M (2009) Cross validation of the Montreal Cognitive Assessment in community dwelling older adults residing in the Southeastern US. Int J Geriatr Psychiatry 24(2):197-201

23. Magierska J, Magierski R, Fendler W, Kłoszewska I, Sobów TM (2012) Clinical application of the Polish adaptation of the Montreal Cognitive Assessment (MoCA) test in screening for cognitive impairment. Neurol Neurochir Pol 46(2):130-139

24. Kim HY (2013) Statistical notes for clinical researchers: assessing normal distribution (2) using skewness and kurtosis. Restor Dent Endod 38(1):52-54

25. Neumann PJ, Araki SS, Gutterman EM (2000) The use of proxy respondents in studies of older adults: lessons, challenges, and opportunities. J Am Geriatr Soc 48(12):1646-1654

26. Sixsmith J, Sixsmith A, Fänge AM et al (2014) Healthy ageing and home: the perspectives of very old people in five European countries. Soc Sci Med 106:1-9

27. Wilson KA, Dowling AJ, Abdolell M, Tannock IF (2000) Perception of quality of life by patients, partners and treating physicians. Qual Life Res 9:1041-1052

28. Pickard AS, Knight SJ (2005) Proxy evaluation of health-related quality of life. Med Care 43(5):493-499

29. Nicholson C, Gordon AL, Tinker A (2017) Changing the way "we" view and talk about frailty. Age Ageing 46(3):349-351

30. Capuzzo M, Grasselli C, Carrer S, Gritti G, Alvisi R (2000) Quality of life before intensive care admission: agreement between patient and relative assessment. Intensive Care Med 26(9):1288-1295

31. Sprangers MA, Aaronson NK (1992) The role of health care providers and significant others in evaluating the quality of life of patients with chronic disease: a review. J Clin Epidemiol 45(7):743-760

32. Layne C (1983) Painful truths about depressives' cognitions. J Clin Psychol 39:848-853

33. Long K, Sudha S, Mutran EJ (1998) Elder-proxy agreement concerning the functional status and medical history of the older person: the impact of caregiver burden and depressive symptomatology. J Am Geriatr Soc 46(9):1103-1111

34. Kellerman SE, Herold J (2001) Physician response to surveys. Am J Prev Med 20(1):61-67

35. Dillon C, Serrano CM, Castro D, Perez Leguizamón P, Heisecke SL, Taragano FE (2013) Behavioral symptoms related to cognitive impairment. Neuropsychiatr Dis Treat 9:1443-1455

36. Davis DHJ, Creavin ST, Yip JLY, Noel-Storr AH, Brayne C, Cullum S (2015) Montreal Cognitive Assessment for the diagnosis of Alzheimer's disease and other dementias (review). Cochrane Database of Syst Rev 10:CD010775 